\title{
Evaluación de las políticas públicas de atención a víctimas que han sufrido explotación infantil en el posacuerdo ${ }^{1}$
}

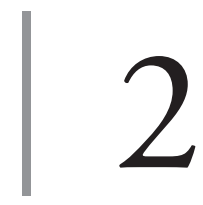

\author{
https://doi.org/10.21830/9789585241480.02
}

Paola Alexandra Sierra-Zamora
Escuela Militar de Cadetes "General José María Córdova"
Ana María Mateus Poveda
Universidad Católica de Colombia
Manuel Bermúdez-Tapiat
Universidad Privada San Juan Bautista
Juan Bernardo Moscoso Martínez"

1 Este capítulo hace parte de los resultados del proyecto de investigación "Desafíos contemporáneos para la protección de derechos humanos en escenarios de posconflicto desde enfoques interdisciplinarios - FASE II" del Grupo de Investigación Persona, Instituciones y Exigencias de Justicia, del Centro de Investigaciones Sociojurídicas, Facultad de Derecho, de la Universidad Católica de Colombia, registrado con el código COL0120899 de Minciencias. También contó con la colaboración de los siguientes proyectos de investigación: "La legitimidad de las Fuerzas Militares en la geopolítica nacional e internacional de Colombia” del Grupo de Investigación en Ciencias Militares, de la Escuela Militar de Cadetes “General José María Córdova” (ESMIC), registrado con el código COL0082556 de Minciencias; "Análisis del perfil criminológico de adolescentes infractores para evaluar tasas de reincidencia delictiva que afecte la seguridad ciudadana”, aprobado por Resolución Vicerrectoral No 058-2018-VRIUPSJB de la Universidad Privada San Juan Bautista, Perú; y "Modelo de enseñanza-aprendizaje en (DDHH) Derechos Humanos y (DIH) Derecho Internacional Humanitario en el Ejército Nacional de Colombia a través del juego y la simulación” del Grupo de Investigación del Centro de Misiones Internacionales y Acción Integral (CEMAI). Los puntos de vista y los resultados de este capítulo pertenecen a los autores y no reflejan necesariamente los de las instituciones participantes.

2 Abogada de la Universidad Católica de Colombia. Magíster en Derechos Humanos, Democracia y Justicia Internacional por la Universitat de València, Espańa. PhD (c) del Programa de Derechos Humanos, Democracia y Justicia Internacional por la Universitat de València, Espańa. Investigadora del Grupo de Investigación en Ciencias Militares de la Escuela Militar de Cadetes "General José María Córdova”. Orcid: https:// orcid.org/0000-0002-3146-7418. Contacto: paola.sierra@esmic.edu.co

3 Estudiante de la Facultad de Derecho de la Universidad Católica de Colombia y miembro del semillero de investigación "Observatorio de justicia constitucional de derechos humanos", vinculado al Grupo de Investigación Persona, Instituciones y Exigencias de Justicia, de la Universidad Católica de Colombia.

4 Abogado (magna cum laude) de la Pontificia Universidad Católica del Perú. Profesor-investigador de la Universidad Privada San Juan Bautista y profesor de la Facultad de Derecho de la Universidad Nacional Mayor de San Marcos. Consultor en temas de reforma del sistema de justicia en el Perú para el Banco Mundial, el Congreso de la República, el Poder Judicial y la Academia de la Magistratura. Orcid: https://orcid.org/0000-0003-1576-9464. Contacto: manuel.bermudez@upsib.edu.pe

5 Capitán del Ejército Nacional de Colombia. Administrador logístico y profesional en Ciencias Militares, de la Escuela Militar de Cadetes "General José María Córdova”. Especialista en Conducción y Administración de Unidades Militares, de la Escuela de Armas Combinadas del Ejército. Investigador del Centro de Misiones Internacionales y Acción Integral (CEMAI) del Ejército Nacional de Colombia. Orcid: https://orcid. org/0000-0001-5633-5633. Contacto: juan.moscosoma@buzonejercito.mil.co 


\section{Resumen}

Una de las situaciones de extrema gravedad durante el periodo de conflicto armado interno en Colombia es la explotación infantil por parte de las guerrillas. Con el foco en este problema social, se evalúan en este capítulo las políticas públicas que se implementarán para atender a las víctimas que se han ubicado e identificado para que, en la etapa del posacuerdo, puedan insertarse en la sociedad. Esta reinserción implica el alivio del dolor y el daño provocados por la lesión de sus derechos humanos, especialmente, en su etapa infantil y adolescente. También se revisan aquí las consecuencias lamentables de estos hechos para una etapa adulta, las secuelas de traumas psicológicos o lesiones corporales que pueden incidir negativamente en su desarrollo social, cultural y económico.

Palabras clave: explotación infantil; interés superior del niño; políticas de Estado; políticas públicas; trabajo infantil; víctima

\section{Introducción}

En Colombia, desde el inicio de las acciones insurgentes ejecutadas por las guerrillas, se ha registrado la vulneración de los derechos humanos de cientos de miles de víctimas, lo cual puede ser enfocado específicamente en las víctimas directas del conflicto armado interno y extensivamente en toda la población colombiana (Garay Acevedo y Pérez Guecha, 2018; Cubides et al., 2019).

Como consecuencia de esta situación se ha generado un problema en la nación, con lo cual se da lugar a un severo cuestionamiento de la legitimidad del Estado por parte de la población, puesto que no se atiende al bien común institucionalizado por la administración pública (Bermúdez-Tapia, 2018; Corcione Nieto et al., 2019) y, en consecuencia, no hay lugar a un acuerdo social ni a sus beneficios (Nasi y Rettberg, 2005). Un "problema-nación” que debe ser conceptualizado identificándose al conflicto armado generado por las guerrillas como

Confrontación existente, por un lado, entre las fuerzas militares del Estado y las agrupaciones armadas que, con relativa unidad de mando, justifican su actuar por la necesidad de una transformación política, social y económica del país . (Contreras, 2003, p. 122)

Tal situación ha exigido que las autoridades políticas del gobierno nacional tengan que buscar un mecanismo idóneo para alcanzar la paz en todo 
el territorio nacional, mecanismo que ha sido ejecutado mediante el proceso del acuerdo de paz (Peña Chivatá, Sierra Zamora y Hoyos Rojas 2019). Sin embargo, los acuerdos de paz como solución positiva de un conflicto armado se refieren a un trato entre las partes que puede requerir de la mediación de un tercero imparcial (Valencia et al., 2012) ya que el acuerdo de paz permite que se superen las diferencias que ocasionan en buena medida el origen del conflicto (Acevedo y Rojas, 2016). Así las cosas, es un mérito que estos conceptos permitan que formalmente exista "un antes y un después" de las acciones provocadas por las FARC-EP en el país.

De este modo, el Acuerdo Final para la Paz (en adelante, AFPP) en Colombia se plantea una serie de puntos a conocer para la corrección y el perfeccionamiento de conductas humanas nocivas para determinadas sociedades (Fernández-Osorio, 2019), como la explotación infantil (en adelante, EI). Esta última se define como el modo en el cual determinadas personas se aprovechan de niños, niñas y adolescentes (en adelante, NNA) para obtener mayores recursos económicos, vulnerando derechos fundamentales no solo de los menores sino también de sus familiares, tales como el derecho a la vida, educación, salud, alimentación y protección.

Teniendo en cuenta lo anterior, el presente capítulo se desarrolla mediante la metodología de investigación de carácter cualitativo (Ruiz, 2012), manteniendo el desarrollo descriptivo, bibliográfico y legislativo, con investigación exploratoria y propositiva, con el objetivo de analizar un aspecto que permite exponer una cuestión en la investigación: ¿Cuáles serán los mecanismos de protección que desarrollará el Estado colombiano para afrontar eficientemente la lucha contra la explotación infantil en el periodo del posacuerdo?

El capítulo se desarrolla en tres ejes temáticos. En una primera parte se pretende dar a conocer algunas generalidades de la EI, incluidas sus variedades, para así poder comprender el alcance de este problema social. En una segunda etapa se enuncian los planes de desarrollo que el acuerdo de paz brinda para las víctimas que han sufrido las consecuencias de un trabajo infantil (en adelante, TI) y, de esta forma, lograr evaluar si este plan se está implementado correctamente. En una tercera etapa se plantea dar a conocer los mecanismos de protección para NNA tales como programas y campañas de erradicación del 
TI, para tener un completo conocimiento de las estrategias de erradicación de problemas socioeconómicos que afectan a una población sensible y en situación de vulnerabilidad. En el desarrollo final del capítulo se exponen las conclusiones.

\section{La explotación infantil como un problema social}

En primera instancia se pretende explicar qué es la EI por medio de diferentes referencias doctrinales para comprender el nivel de incidencia social de este problema y, a su vez, identificar los derechos fundamentales vulnerados de los NNA afectados. La explicación dogmática permite exponer los diferentes tipos de explotación que existen, porque estos pueden diferir en función de la realidad en evaluación. Por lo anterior, solo nos centraremos en los hechos provocados por las acciones insurgentes en Colombia que han afectado a la población infantil, sometiéndola a acciones de explotación de naturaleza social, económica y laboral (Cabrera Cabrera et al., 2018; Castro Torres, Casallas Torres y Salgado Torres, 2019). Para empezar, es importante tener en claro que, en la evaluación teórica, la EI se asocia a

\footnotetext{
Una disfuncionalidad en la que los niños asumen roles que no les corresponden y que afectan su adecuado desarrollo, inmiscuyéndose (interviniendo) en condiciones impropias para su edad y alejándose del entorno escolar, familiar y social propios para su evolución física y sicológica. Este fenómeno amenaza el bienestar generacional de la sociedad, ya que reproduce la pobreza y es un detonante de la inclusión social. (Bohórquez, 2009, p. 2)
}

Teniendo en cuenta la anterior definición de EI, se puede decir que este fenómeno social tiene un nivel altamente perjudicial para los NNA, ya que afecta el desarrollo psicológico y físico de un menor de edad (Bermúdez-Tapia, 2012). Tal situación puede ser provocada por los siguientes actores:

a. Los progenitores, quienes obligados por sus condiciones económicas negativas pueden forzar a ejecutar trabajos ajenos a la realidad mental y física de sus propios hijos. 
b. Los familiares, principalmente, cuando existe la ausencia de los progenitores en forma inmediata, oportuna o constante.

c. Uno de los progenitores, principalmente, a consecuencia de una situación de violencia económica y familiar impuesta a la familia por parte de quien ejecuta la explotación infantil.

d. Agentes externos al entorno familiar, como el que se evalúa en el este capítulo, en particular, porque se identifica las FARC-EP como uno de los principales agentes que ha ejecutado acciones de EI a NNA en el país.

Los NNA necesitan de una vida, en la medida de lo posible, estable y con la posibilidad de desarrollar sus expectativas en forma positiva en un esquema físico, psicológico, social, cultural y económico, para así poder incorporarse como fuerza aportante a la población del país.

El proceso productivo en lo económico y en lo laboral, sin embargo, no ha tomado en cuenta que los NNA no forman parte de este universo, que exige la participación de personas formadas, capaces y con un nivel de discrecionalidad en la disposición de sus derechos. Por tanto, es posible observar situaciones en las cuales menores de edad son sometidos a actividades que en esencia les resultan desproporcionadas, lesivas y limitativas.

Según la Organización Internacional del Trabajo la EI es todo trabajo que priva a los niños de su niñez, su potencial y su dignidad. Esta situación es perjudicial para su desarrollo físico, mental, moral y psicológico (Soto, 2012, p. 1), pues interfiere con su escolarización, lo priva de la posibilidad de asistir a clase, los obliga a abandonar la escuela de forma prematura o les exige combinar el estudio con un trabajo pesado que consume mucho tiempo y energía.

Las dos definiciones dadas de EI son complementarias entre ellas, ya que evidencian el gran daño que genera a los NNA, el cual puede exponerse en tres niveles:

a. En lo psicológico, por cuanto los obliga a llevar a cabo acciones ajenas a su libre desarrollo y contexto temporal en función a su edad.

b. En lo físico, debido principalmente a que la mayoría de las actividades a las cuales son sometidos exigen una actividad física extraordinaria para su rango de edad. 
c. En lo social, económico y moral, porque, a pesar de verse involucrados en un contexto productivo, los resultados de sus actividades les son ajenos. Eventualmente este factor es el que provoca una mayor situación negativa, porque puede provocar la explotación sexual, el tráfico humano ilegal o la extorsión a los progenitores y familiares, hecho que evidencia el contexto de incidencia social.

Sobre la base de estos elementos, el Plan Nacional de Acción para la Erradicación del Trabajo Infantil en Colombia define en forma amplia algunas situaciones que conviene tener presentes:

a. La esclavitud o prácticas análogas a la esclavitud, como la venta y el tráfico de niñas y niños, la servidumbre por deudas y la condición de empleado vinculado a un trabajo forzoso u obligatorio.

b. La utilización, el reclutamiento o la oferta de niñas y niños para la pornografía o la explotación sexual de cualquier clase.

c. La utilización, el reclutamiento o la oferta de niñas y niños para la realización de actividades ilícitas, en particular, la producción y tráfico de estupefacientes, tal como se define en los tratados internacionales pertinentes. En este punto, Bermúdez-Tapia menciona que en el Perú se ha podido observar que grupos vinculados al narcoterrorismo emplean a menores de edad para la ejecución de sus actividades ilícitas, especialmente porque al ser inimputables no son perseguidos por las autoridades policiales y judiciales, hecho que permite su reinserción en la cadena productiva del narcotráfico en el país (2019, p. 11).

d. El reclutamiento forzado de menores de edad para formar parte de los grupos insurgentes. Esta fue la acción más perjudicial provocada por las FARC-EP en sus actividades, porque amplió el daño a una población ajena a sus propios lineamientos de "lucha contra el Estado", lo que no es legítima ni eficaz, por cuanto al final convirtió a NNA en las primeras víctimas en los enfrentamientos con las Fuerzas Militares, dada la inexperiencia y, sobre todo, el miedo que los menores mostraban en estas situaciones. 


\subsection{La afectación a los derechos de los NNA por la EI}

Es muy importante identificar el nivel de afectación de (a) los derechos humanos, (b) los derechos fundamentales y (c) los derechos económicos, sociales y culturales de los NNA como consecuencia de actos vinculados a la EI. Este contexto permite identificar de forma genérica la "afectación a los derechos" de los NNA, porque el desarrollo programático de derechos humanos y derechos fundamentales, de acuerdo con la legislación nacional e internacional aplicable a nuestra evaluación, indica dos aspectos centrales relacionados:

a. El carácter efectivo que implica la tutela de un "tipo de derecho" en casos especiales en los cuales la persona a quien se le debe evaluar un daño es menor de edad.

b. El ámbito especial denotado por la legislación, porque los "derechos fundamentales" son aludidos por el constituyente nacional, que difiere del sentido extensivo de los "derechos humanos" desarrollados, sobre todo, en el ámbito del derecho internacional.

De este modo, es posible evaluar los aspectos estructurales de este estudio, los cuales permitirán un análisis mucho más práctico al momento de evaluar la EI que ha afectado a los NNA durante el periodo del conflicto armado interno.

Bajo este parámetro referencial, los derechos humanos son aquellos elementos imprescriptibles, inalienables, irrenunciables y universales (Gálvez, 2014) que dotan a toda persona de un conjunto de elementos que deben ser tutelados por el Estado, especialmente, en mérito a su obligación de cumplir un compromiso internacional asumido voluntariamente con base en su soberanía.

En cambio, los derechos fundamentales son aquellos que imponen al Estado la ejecución de acciones complementarias a la tutela de derechos humanos, conforme a lo expuesto en el párrafo precedente. Además, se desarrollan en la legislación nacional y guían a los órganos jurisdiccionales para valorar las acciones necesarias y suficientes que se requieran para tutelar un derecho en forma inmediata y eficaz (Bermúdez-Tapia, 2007).

De lo detallado preliminarmente, la Constitución Política identifica los principales derechos que todo NNA debe tener: la vida, la integridad física, 
la salud y la seguridad social; la alimentación equilibrada, su nombre y nacionalidad; tener una familia y no ser separados de ella; el cuidado y el amor; la educación, la cultura, la recreación y la libre expresión de su opinión.

Tal referencia normativa permite detallar los espacios de contradicción que termina provocando el hecho de que la EI no vulnera todos los derechos mencionados anteriormente, pero sí los más importantes según el Fondo de las Naciones Unidas para la Infancia (Unicef), los cuales son:

a. Derecho a la vida en un ambiente equilibrado. En este punto, se destaca que, por las condiciones extremas a las cuales son sometidos los NNA en situación de EI, la salud y las condiciones de desarrollo psicológico y físico resultan afectadas de modo progresivo y pueden constituir un severo problema en el propio desarrollo de vida de una persona, en su etapa de nińo, adolescente o adulto.

b. Derecho a la educación. Es uno de los derechos más importantes en la etapa de desarrollo hacia la adultez de todo niño y adolescente, porque en este periodo de vida es cuando se requiere de un proceso constante y progresivo de aprendizaje.

Este derecho se complementa con el derecho a la recreación, ya que en un espacio como la escuela los NNA inician un proceso de formación para su personalidad, su autoestima y carácter por medio de relaciones sociales.

Cuando los menores de edad trabajan desde una corta edad es más difícil obtener conocimientos básicos, lo cual los condiciona a un rango económico y social limitado y negativo, además de generar elementos psicológicos perjudiciales al provocar situaciones de autoexclusión social.

c. Derecho a la integridad personal. Los NNA deben estar protegidos de todo maltrato, tanto psicológico como físico que otra persona pueda cometer hacia ellos. En este ámbito, la integralidad está vinculada a un factor de edad y esto permite identificar el nivel de daño provocado por parte de cualquier agente que desarrolle acciones propias de la EI. 
d Derecho a todo lo que requieren los NNA para su desarrollo integral, como la alimentación, vestido, educación y salud. Cuando los NNA son expuestos a casos de EI es posible que no gocen en la totalidad de lo anteriormente nombrado, puesto que su vida está encaminada única y exclusivamente al trabajo.

La Convención Internacional sobre los Derechos del Niño reafirma que los NNA tienen iguales derechos que todas las personas; especifica estos derechos para las particularidades de la vida y madurez de los niños; establece derechos propios de los niños; regula los conflictos jurídicos derivados del incumplimiento de estos o de su colisión con los derechos de los adultos; y orienta y limita actuaciones de las autoridades públicas y las políticas públicas en relación con la infancia (Cillero, 1999).

El TI, por ejemplo, se da por la pobreza, con lo cual los menores se ven forzados a colaborar a sus familias exponiéndose a un grado de peligro muy alto (Pautassi y Zibecchi, 2010).

\subsection{Las peores situaciones en las que se identifica el trabajo o explotación infantil}

La EI es un fenómeno mundial, porque las sociedades no suelen tomar en cuenta el contexto que rodea a los NNA, en particular, cuando surgen situaciones de extrema violencia social o política en el país. Por ello es que el registro de EI o de TI en el mundo son usuales en el ámbito de las indagaciones sobre la temática. Consecuentemente, la EI y el TI están prohibidos por todas las legislaciones, sin que ello implique la total defensa de los derechos de estos menores de edad que lamentablemente están expuestos a una serie de factores que provocan su inserción en tales situaciones.

Según el Convenio sobre las Peores Formas de Trabajo Infantil (Organización Internacional del Trabajo [OIT], 2001), estas son las siguientes:

a) Todas las formas de esclavitud o las prácticas análogas de esclavitud, como la venta y el tráfico de niños, la servidumbre por deudas y la condición de siervo, y el trabajo forzoso u obligatorio, incluido el reclutamiento forzoso u obligatorio de niños para utilizarlos en conflictos armados. 
b) La utilización, el reclutamiento o la oferta de niños para la prostitución, la producción de pornografía o actuaciones pornográficas.

c) La utilización, el reclutamiento o la oferta de niños para la realización de actividades ilícitas, en particular la producción y el tráfico de estupefacientes, tal como se definen en los tratados pertinentes.

d) El trabajo que, por su naturaleza o por las condiciones en que se lleva a cabo, es probable que dañe la salud, la seguridad o la moralidad de los niños.

\subsection{Los tipos de trabajo infantil}

Es “toda actividad física o mental, remunerada o no, dedicada a la producción, comercialización, transformación venta o distribución de bienes o servicios, realizada en forma independiente o al servicio de otra persona natural o jurídica, (...) por persona menor de 18 años” (OIT y IPEC, 2003, p. 24) resulta ser negativa o perjudicial, porque, en esencia, se debe detallar las situaciones diferenciales entre los elementos en evaluación:

1. Existen algunas labores que no resultan exigentes ni perjudiciales, y que pueden ser desempeñadas por los menores de edad. En este ámbito, se registran dos subniveles:

i. El trabajo vinculado al ámbito familiar, en particular, el que se desarrolla para colaborar con los padres, lo que incluye la ejecución de acciones que implican un esfuerzo físico y psicológico, como el desarrollo de los deberes básicos de la casa.

ii. El trabajo rentable, que está regulado por la legislación tanto nacional como comparada y que impone algunas condiciones como la dirección y acción de los progenitores en el control de las actividades a ser desarrolladas, el desarrollo libre y voluntario de dichas acciones en el ámbito laboral por parte de los NNA, la no exclusión de las actividades propias de la edad y el desarrollo formativo personal, social, cultural y humano de todo NNA.

De esta forma, es posible detallar que no todo TI implica una situación de EI, pero en el presente caso solo haremos la referencia a las situaciones gene- 
radas en el conflicto armado interno que involucran el nivel de EI ejecutado contra NNA a manos de los grupos insurgentes.

A partir de lo anteriormente expuesto, se amplía el ámbito normativo y, por ello, la Constitución Política y los tratados internacionales instauran rangos o valores para el ingreso a una actividad laboral con base en una edad de referencia para acceder a la vida productiva. Los NNA pueden ingresar a la vida laboral en los denominados "trabajos ligeros", que no afectan su salud ni su vida cotidiana. Los principales son los siguientes —en ellos es posible identificar aspectos positivos y negativos-

a. Trabajo doméstico: Muy común y en ocasiones considerado como aceptable. Tiene lugar fuera y dentro del hogar familiar. Cuando es fuera, las jornadas suelen ser largas, los NNA no pueden asistir a la escuela y están lejos de amigos o familiares, lo cual genera una situación prohibida por la legislación, que puede deberse a factores familiares, sociales y económicos que involucran a los progenitores en la toma de decisiones negativas contra sus propios hijos.

b. Labores agrícolas: La mayoría de los niños que están vinculados a condiciones laborales o económicas productivas, trabajan en labores agrícolas en zonas rurales.

c. Trabajo en industrias: Puede ser regular o casual. Incluye actividades como tejer alfombras, pulir piedras preciosas, fabricación de prendas de vestir, sustancias químicas, fuegos artificiales y fósforos, entre otros. Todo ello puede ocasionar envenenamiento, daños a la vista, oído, cortes, quemaduras e incluso la muerte en los NNA.

d. Trabajo en minas y canteras: Los niños trabajan largas jornadas sin contar con la protección o la formación adecuadas. Padecen de agotamiento físico, fatiga y desórdenes en el sistema muscular y óseo.

e. Esclavitud y trabajo forzoso: Se incluye en esta categoría lo que se conoce como servidumbre por deudas, más común en áreas rurales. Esta forma de EI está vinculada con la opresión de minorías étnicas o pueblos indígenas. Los niños también son reclutados a la fuerza como soldados o para trabajar para el ejército en zonas de conflicto.

f. Prostitución y trata de menores: Esta es una de las peores formas de trabajo infantil. Los peligros a los cuales se enfrentan los NNA son extremos y van desde la degradación moral hasta las enfermedades de transmisión sexual, embarazos no deseados e incluso la muerte.

g. Trabajo en la economía informal: Incluye toda una serie de actividades como limpiar zapatos, mendigar, conducir o recoger basura, entre otras. (CSI, 2008) 


\subsection{La EI ejecutada por las FARC-EP}

Dado que este texto desarrolla el contexto del posacuerdo en Colombia, es pertinente hacer la evaluación del contexto temporal en el que se han podido ubicar referencias de EI provocada por grupos insurgentes durante el conflicto armado interno (Fernández-Osorio y Pachón Pinzón, 2019). En este sentido, los grupos insurgentes, usualmente han ejecutado acciones de EI identificadas de la siguiente manera:

a. La captura de NNA para someterlos a actividades productivas a favor de sus intereses. En este caso, los NNA han sido expuestos a una serie de situaciones como el desarrollo de trabajos domésticos, explotación sexual infantil o el desarrollo de actividades de naturaleza ilícita para generar una rentabilidad económica a favor de los captores.

b. La ejecución de actividades de naturaleza económica bajo subordinación sin provocar una condición negativa en el desarrollo físico del NNA, en particular, cuando los empleaban en actividades agrícolas en las zonas administradas por grupos insurgentes.

c. La inclusión de NNA en las acciones de naturaleza armada y beligerante de los grupos insurgentes (aspecto analizado en el capítulo precedente).

\section{El posacuerdo en Colombia}

Colombia es un país que a lo largo del tiempo ha presentado diversos problemas y enfrentamientos con grupos guerrilleros, en especial, con las Fuerzas Armadas Revolucionarias de Colombia (FARC), que tenían como propósito la toma del poder político del país, en conjunción con la inconformidad y la rebeldía de las grandes masas de desposeídos del campo y la ciudad. Su primera declaración política se conoce como Programa Agrario de los Guerrilleros y en él se expresa que se alzan en armas porque en el país están cerradas las vías de la lucha política legal, pacífica y democrática (FARC-EP, 2019). 
A raíz de varias problemáticas provenientes de las FARC — desapariciones, asesinatos, violaciones y desplazados, entre otras_- , han quedado aproximadamente 7 millones de víctimas en más de cincuenta años de conflicto (Sánchez, 2019). Evidenciada esta situación, se decide empezar con un proceso de paz con el cual siempre será muy importante, más allá de lo político y lo legal, la construcción de un consenso social en torno al proceso que permita tener un respaldo sobre su desarrollo y sus posteriores consecuencias (o posconflicto) (Cárdenas, 2013). Un proceso de paz debe estar 100\% vigilado para que se cumpla lo que se tiene acordado desde un principio. Variedad de presidentes de Colombia han buscado llegar a un acuerdo de paz con las FARC, pero aquel que lo logró fue Juan Manuel Santos, durante su periodo presidencial en el año 2016.

El acuerdo de paz tiene como propósito terminar el conflicto. A grandes rasgos, este pretende, en primera instancia, que los habitantes del campo tengan tierra, tengan cómo producir y vivir, y también que participen en la planeación de sus regiones. En segunda instancia, busca que haya más voces diversas en la política, que más ciudadanos participen en asuntos públicos y que no se vuelvan a mezclar las armas y la política. En tercera instancia, busca que las FARC dejen sus armas, que sus exintegrantes se reincorporen a la vida civil y que haya garantías de seguridad para todos.

Además, se plantea una solución al cambiante problema de las drogas ilícitas (Niño González, 2016) y se busca que, a través de los mecanismos y medidas que integran el Sistema Integral de Verdad, Justicia, Reparación y No Repetición (SIVJRNR), se logre una reparación de víctimas y que se vislumbre una verdad sobre lo ocurrido, entre otras. Por último, que el AFPP sea acogido por todos los colombianos, que tenga un acompañamiento internacional y que se implemente y se haga un seguimiento a ese proceso (Oficina del Alto Comisionado para la Paz, 2016, pp. 4-5).

\subsection{La vida de los NNA durante el conflicto armado}

Los NNA que han sido víctimas del conflicto armado proveniente de las FARC, por lo general, son hijos de padres campesinos. Ellos han sido afectados directamente por la violencia en sus regiones; su sentido de realidad gira 
entorno a la supervivencia en un marco de conflicto que limita los derechos más básicos.

En la mayoría de los casos, los NNA se han visto obligados a abandonar la escuela para ayudar económicamente a su familia, también por causa del conflicto armado o porque han sido reclutados.

En sus zonas de habitación, los niños y niñas padecen restricción en el ingreso de alimentos (57\%), 27 están expuestos a los combates permanentes (84\%), las bombas (43\%), las minas antipersona, los atentados, las amenazas, los secuestros, la extorsión que afecta el comercio, el gasto y el consumo (86\%), y la destrucción de sus escuelas, de los centros de salud y de infraestructura asistencial y de servicios básicos (92\%). (Springer, 2012, pp. 20-21)

Las problemáticas nombradas anteriormente causan un nivel total de vulneración a los derechos de los NNA, ya que quedan sometidos a unas actividades en las que lo único que les queda por hacer es usar armas, dejar muertos y desaparecidos. Estas actividades son increíbles al momento de saber que los NNA son sus coautores a causa del reclutamiento.

\subsubsection{El ReClutamiento DE NNA}

Un factor principal para hacer posible el reclutamiento de NNA es la capacidad que tienen los integrantes del grupo guerrillero de las FARC para que otros hagan su voluntad, ya sea por medio de falsas promesas, mentiras o amenazas, entre otras. Conductas difíciles de evitar y rechazar, puesto que los menores por lo general se dejan influenciar y creen que todo lo que les dicen las demás personas está bien y es correcto para ellos.

Se ha logrado evidenciar, efectivamente, que los NNA no se vinculan a estos grupos guerrilleros por su propia voluntad, ya que tienen un amplio conocimiento en cuanto a lo que se debe realizar una vez integrados a sus filas, por esta razón se recurre al reclutamiento forzado. Para mayor claridad, el reclutamiento se puede definir de siguiente manera:

Es un acto de fuerza, facilitado por la vulnerabilidad social y económica de los afectados, pero que, de ninguna manera, tendría lugar sin la existencia de un conflicto armado, cuya violencia produce dinámicas que alienan todos los derechos y las libertades de las comunidades sometidas y arrastra consigo, especialmente, a los más vulnerables. (Springer, 2012, p. 31) 
Es muy fácil lograr que los NNA se integren a los grupos guerrilleros, ya que, por lo general, son pobres, no tienen la posibilidad de estudiar y cuentan con tiempo disponible. En la mayoría de los casos, el reclutamiento de NNA se debe a una serie de características sociofamiliares que rodean a este grupo poblacional y que permiten el desarrollo de actividades que resultan óptimas y eficientes para los intereses de los grupos insurgentes. Esto es así porque los menores de edad presentan las siguientes características:

a. No tienen pareja.

b. Por lo general no tienen hijos ni personas a su cargo.

c. Soportan de manera efectiva actividades intensas como largas caminatas, el desgaste de huir y el esfuerzo físico.

d. No presentan un cuadro de salud que registre enfermedades con regularidad.

e. No tienen el conocimiento cabal de sus derechos, lo que los convierte en presa fácil para las acciones y condicionamientos laborales, por ejemplo, no cuentan con remuneración en contraprestación de las labores ejecutadas, sino que solo en ocasiones muy contadas se le asignaban pequeñas compensaciones, especialmente, para generarles una mayor vinculación a sus actividades.

f. Son moldeables psicológicamente para hacer lo que los demás ordenen.

g. Los más pequeños realizaban la provisión de alimentos, transporte de mensajes, documentos y comunicaciones.

h. Son personas ajenas a las acciones represivas y punitivas del Estado, en particular, por su condición de inimputabilidad, que les permiten a quienes ejecutan EI el desarrollo de crímenes con mayor facilidad o la ejecución de actos que sostienen en forma secundaria sus acciones ilegales.

El reclutamiento, entonces, es una práctica que compromete múltiples violaciones a los derechos humanos y que, no obstante, es ejecutada de manera sistemática y con la clara intención de someter a una población altamente vulnerable. De convertir a las víctimas en victimarios (Roldán, 2002). 
Existen tres fases en el proceso de reclutamiento:

a. Adaptación: consta de largas conversaciones (incluso les entregan pequeñas cantidades de dinero para enviar a sus familias).

b. Adoctrinamiento y endurecimiento: se pretende, en primera instancia, inculcar los ideales de la guerra en los NNA y, en segundo lugar, generar un proceso de deshumanización y despersonalización.

c. Adiestramiento: se les enseńa a defenderse y a combatir para enfrentarse a la guerra.

El reclutamiento consta de todo lo anteriormente mencionado y, a la final, solo genera daños tantos físicos como mentales, no solo a los NNA sino a sus familiares.

\section{La ubicación de los NNA en el AFPP}

En el AFPP se plantean unos acuerdos con la finalidad de reconocer los derechos de las personas y garantizar una paz plena. Si se revisa este punto con la atención puesta en los NNA, se encuentran algunos elementos generales (Oficina del Alto Comisionado para la Paz, 2016, pp. 23-207):

a. Que la Reforma Rural Integral (RRI) en materia de alimentos y nutrición pretende asegurar para toda la población rural y urbana en Colombia la disponibilidad y el acceso suficiente en oportunidad, cantidad, calidad y precio a los alimentos necesarios para una buena nutrición. En especial, niños y niñas, mujeres gestantes y lactantes y personas adultas mayores deben contar con esta posibilidad, para quienes se promueve de forma prioritaria la producción de alimentos y la generación de ingresos.

La política de desarrollo agrario integral es universal y su ejecución pone en primer lugar la población y los territorios más necesitados y vulnerables, así como las comunidades más afectadas por la miseria, el abandono y el conflicto, con énfasis en pequeños y medianos productores y productoras. Especial atención merecen los derechos de las víctimas del conflicto, de los niños y niñas, de las mujeres y de los adultos mayores.

b. El objetivo de los Programas de Desarrollo con Enfoque Territorial (PDET) es lograr la transformación estructural del campo y el ámbito rural, así como un relacionamiento equitativo entre el campo y la ciudad. 
De esta manera, se espera asegurar el bienestar y el buen vivir de la población en zonas rurales, con el fin de que también se hagan efectivos sus derechos políticos, económicos, sociales y culturales, y se logre revertir los efectos de la miseria y el conflicto.

c. El objetivo central de los planes nacionales para la Reforma Rural Integral es, por una parte, la superación de la pobreza y la desigualdad para alcanzar el bienestar de la población rural y, por otra, la integración y el cierre de la brecha entre el campo y la ciudad. De conformidad con el acuerdo de paz, las autoridades competentes deberán elaborar y poner en marcha los Planes Nacionales en el territorio. La superación de la pobreza no se logra simplemente mejorando el ingreso de las familias, sino asegurando que niños, niñas, mujeres y hombres tengan acceso adecuado a servicios y bienes públicos.

En educación rural, con el propósito de brindar atención integral a la primera infancia; garantizar la cobertura, la calidad y la pertinencia de la educación; erradicar el analfabetismo en las áreas rurales; promover la permanencia productiva de los y las jóvenes en el campo; y acercar las instituciones académicas regionales a la construcción del desarrollo rural, el gobierno nacional creará e implementará el Plan Especial de Educación Rural, para cuyo desarrollo se tendrán en cuenta los siguientes criterios:

i. La cobertura universal con atención integral a la primera infancia.

ii. Modelos flexibles de educación preescolar, básica y media, que se adapten a las necesidades de las comunidades y del medio rural, con un enfoque diferencial.

iii. La construcción, reconstrucción, mejoramiento y adecuación de la infraestructura educativa rural, incluyendo la disponibilidad y permanencia de personal docente calificado y el acceso a tecnologías de información.

iv. La garantía de la gratuidad educativa para educación preescolar, básica y media.

v. El mejoramiento de las condiciones para el acceso y la permanencia en el sistema educativo de niños, niñas y adolescentes a través de un acceso gratuito a útiles, textos, alimentación escolar y transporte.

vi. La oferta de programas e infraestructura de recreación, cultura y deporte.

vii. La incorporación de la formación técnico-agropecuaria en la educación media (décimo y once).

viii. La implementación de un programa especial para la eliminación del analfabetismo rural. 
d. El desarrollo de programas contra el hambre y la desnutrición con cobertura nacional, especialmente para la población rural en condiciones de miseria, las mujeres gestantes y lactantes, niños y niñas, y para la tercera edad. Estos programas incluirán planes de choque para la población rural más vulnerable y en pobreza extrema.

e. Los menores de edad que hayan salido de los campamentos de las FARC-EP desde el inicio de las conversaciones de paz, así como los que salgan hasta la finalización del proceso de la dejación de armas, serán objeto de medidas de especial atención y protección, que incluirán los principios orientadores del Programa Especial conforme a lo establecido en el Comunicado Conjunto No. 70, de fecha 15 de mayo de 2016, para garantizar la restitución de sus derechos con enfoque diferencial, priorizando su acceso a la salud y a la educación.

A estos menores de edad se les reconocerán todos los derechos, beneficios y prestaciones establecidos para las víctimas del conflicto, así como los derivados de su proceso de reincorporación en los términos contemplados en el acuerdo final y se priorizará su reagrupación familiar cuando ello sea posible, así como su ubicación definitiva en sus comunidades de origen o en otras de similares características, siempre teniendo en cuenta el interés superior del niño.

El seguimiento a estos programas se efectuará por el Consejo Nacional de Reincorporación en articulación con las entidades del Estado competentes y con el apoyo de organizaciones sociales o especializadas encargadas de hacer la veeduría en los términos del Comunicado Conjunto No. 70. La elaboración del Programa Especial de Reincorporación para menores deberá realizarse por parte del Consejo Nacional de Reincorporación en un máximo de 15 días a partir de la firma del Acuerdo Final, con base en la propuesta presentada por parte de la mesa técnica creada mediante Comunicado Conjunto No. 70.

Una vez aprobado el Programa, el Gobierno Nacional tramitará los ajustes normativos que sean necesarios para garantizar su implementación, siempre teniendo en cuenta el interés superior del niño y el Derecho Internacional Humanitario. El Programa deberá garantizar la reincorporación integral del menor y su acompañamiento psicosocial, con la veeduría de las organizaciones sociales o especializadas en los términos del Comunicado Conjunto No. 70, así como también su ubicación en lugares transitorios de acogida en municipios cercanos a las ZVTN, garantizando el derecho a la información de todos los participantes, en especial a los niños, niñas y adolescentes.

f. Enfoque de género: Se pondrá especial énfasis en la protección de mujeres, niñas, niños y adolescentes, quienes han sido afectados por las organizaciones criminales objeto de este acuerdo. Este enfoque tendrá en 
cuenta los riesgos específicos que enfrentan las mujeres contra su vida, libertad, integridad y seguridad y serán adecuadas a dichos riesgos.

g. Unidad Especial de investigación para el desmantelamiento de las organizaciones y conductas criminales responsables de homicidios y masacres, que atentan contra defensores/as de derechos humanos, movimientos sociales o movimientos políticos o que amenacen o atenten contra las personas que participen en la implementación de los acuerdos y la construcción de la paz, incluyendo las organizaciones criminales que hayan sido denominadas sucesores del paramilitarismo y sus redes de apoyo.

Esta Unidad tendrá como base de funcionamiento la articulación de un enfoque investigativo multidimensional que se ocupe de toda la cadena criminal de las organizaciones y conductas objeto de su mandato, incluyendo las conductas criminales que afecten a mujeres, niñas, niños y adolescentes.

Implementará planes metodológicos de investigación especializados respecto a los más graves hechos de victimización que las organizaciones y conductas objeto de este acuerdo ejecuten contra las mujeres, nińas, niños, adolescentes y población LGTBI.

h. Plan de atención inmediata y desarrollo de proyectos productivos a la población escolar: con el fin de: "Mejorar la seguridad alimentaria de las niñas y niños en edad escolar”.

Promover su permanencia en las escuelas se desarrollará un programa de construcción y dotación de comedores escolares y suministro de víveres para garantizar los desayunos a toda la población escolar de los territorios afectados por cultivos de uso ilícito.

i. Cronogramas, metas e indicadores:

Los planes integrales de sustitución y desarrollo alternativo, como los Acuerdos deberán incluir cronogramas de ejecución con metas e indicadores incluyendo los compromisos adquiridos por la comunidad, que permitan medir el impacto de los proyectos en las condiciones de bienestar de las comunidades (niños y niñas, hombres y mujeres) y hacer seguimiento al plan.

j. Programas de Prevención del Consumo y Salud Pública:

El consumo de drogas ilícitas es un fenómeno de carácter multicausal generado por condiciones económicas, sociales, familiares y culturales propias de la sociedad o el medio en el que se desenvuelven las personas que debe ser abordado como un asunto de salud pública.

La solución requiere del compromiso y el trabajo conjunto entre las autoridades, la comunidad y la familia en torno a una política de promoción en salud, prevención, atención integral6 e inclusión social, con especial énfasis en niños, niñas y adolescentes. 
k. Acciones para la promoción en salud y prevención del consumo basadas en la evidencia que respondan a las particularidades y niveles de riesgo de cada territorio y que permitan el fortalecimiento de los factores de protección (apoyo psicosocial, autoestima, solución de conflictos, manejo del tiempo libre, fortalecimiento de núcleos familiares, promoción del compromiso escolar, estilos de vida saludables, desarrollo de habilidades culturales y deportivas y actividades de recreación) frente a los riesgos identificados. Se dará especial atención a la prevención en niños, niñas y adolescentes.

1. Acciones desde el gobierno, las familias, las comunidades y la comunidad educativa para proteger a los niños, niñas y adolescentes del consumo de drogas ilícitas.

m. El Sistema Integral tiene un enfoque diferencial y de género, que se ajusta y responde a las características particulares de la victimización en cada territorio y cada población, y en especial a las necesidades de las mujeres y de los niños y las niñas.

n. Enfoque territorial, diferencial y de género:

Mediante el tratamiento diferenciado de territorios y poblaciones, en especial de las víctimas mujeres, de los niños y las niñas, y de las poblaciones y los colectivos más humildes y vulnerables, y por tanto más afectadas por el conflicto.

o. La Comisión hace parte del Sistema Integral de Verdad, Justicia, Reparación y No Repetición acordado para satisfacer los derechos de las víctimas, terminar el conflicto y alcanzar la paz.

La Comisión deberá cumplir tres objetivos fundamentales, pero únicamente se nombrará el relacionado con NNA, que en su conjunto contribuyen a la no repetición del conflicto:

La Comisión deberá contribuir al esclarecimiento de lo ocurrido, de acuerdo con los elementos del mandato que se describen más adelante, y ofrecer una explicación amplia de la complejidad del conflicto, de tal forma que se promueva un entendimiento compartido en la sociedad, en especial de los aspectos menos conocidos del conflicto, como el impacto del conflicto en los nińos, niñas y adolescentes y la violencia basada en género, entre otros.

p. La Comisión tendrá como mandato esclarecer y promover el reconocimiento del impacto humano y social del conflicto en la sociedad, incluyendo el impacto sobre los derechos económicos, sociales, culturales y ambientales, y las formas notorias en las que el conflicto afectó a las mujeres, a los niños, niñas, adolescentes, jóvenes y adultos mayores.

q. El funcionamiento del componente de justicia dará énfasis a las necesidades de las víctimas mujeres, niñas y niños, quienes sufren de una 
manera desproporcionada y diferenciada los efectos de las graves infracciones y violaciones cometidas con ocasión del conflicto.

Las reparaciones deben responder al llamado de las Naciones Unidas que todo acuerdo de paz debe adoptar un enfoque de género, reconociendo las medidas de reparación y restauración, el sufrimiento especial de las mujeres, y la importancia de su participación y equitativa en el componente de justicia del SIVJRNR.

r. Mediante el reconocimiento de lo ocurrido en el marco del conflicto y del esclarecimiento y rechazo de las graves violaciones a los derechos humanos y de las graves infracciones al Derecho Internacional Humanitario, incluyendo aquellas que han sido históricamente menos visibles como las cometidas contra las mujeres y los niños, las niñas y adolescentes, así como el rechazo a la violencia contra colectivos, movimientos sociales y sindicales, y partidos políticos, en especial los de oposición que fueron severamente victimizados, para que sea un propósito compartido de la sociedad que esto nunca se vuelva a repetir.

De este modo, el fin del conflicto constituye la mejor oportunidad para materializar los derechos de las víctimas a la verdad, la justicia, la reparación y la no repetición, y en general asegurar la satisfacción plena de los derechos humanos de todos y todas, incluyendo los de las mujeres, los niños, niñas, adolescentes, jóvenes y adultos mayores, de las personas en situación de discapacidad, los pueblos indígenas, las comunidades campesinas entre otras, lo cual implica además la adopción de medidas de acción afirmativa, para garantizar de manera plena los derechos de quienes han sido más afectados por el conflicto. La paz como derecho fundamental de todos los ciudadanos es condición necesaria para el ejercicio y disfrute de todos los demás derechos.

En la implementación de todo lo acordado se garantizará el interés superior de los niños, niñas y adolescentes, así como sus derechos y su prevalencia sobre los derechos de los demás.

Hecho el recorrido de referencias a los NNA junto con sus derechos en el AFPP, se evidencia toda la afectación ocasionada por el conflicto para distintos grupos sociales y territorios. El acuerdo inicia la búsqueda de un bienestar social como valor, como aspiración humana, es una cuestión que no tiene nada que ver con las diferencias culturales ni políticas.

Si se aplica la referencia general de que "cualquier persona" debe tener suficiente para comer, agua potable, una educación, asistencia médica, medios de transporte y los servicios públicos básicos, quedaría pendiente referirse a quién y cómo se regula la distribución de estos insumos, y si se provee o se paga 
el bienestar (Gaitán, 2005), cuya equidad generaría tranquilidad en el territorio colombiano, en especial, para quienes han tenido menores posibilidades de acceder a estos "privilegios".

\section{Los mecanismos de protección de NNA frente a la EI}

En el AFPP, sección de Formalización Laboral Rural y Protección Social, se dice que el gobierno nacional fortalecerá al máximo el sistema de protección y seguridad social de la población rural, con un enfoque diferencial (Novoa, 2017) y teniendo en cuenta la situación particular de las mujeres.

A luz de las normas de la OIT, de las que Colombia es parte, y con el propósito de garantizar el trabajo digno, los derechos de los trabajadores y trabajadoras del campo, y su protección social, el gobierno nacional creará e implementará el Plan Progresivo de Protección Social y de Garantía de los Derechos de los Trabajadores y Trabajadoras Rurales. Para su desarrollo se tendrán en cuenta especialmente la erradicación del TI y la adopción de medidas inmediatas para la erradicación de sus peores formas (a las que se aludió en la sección 1.1 de este capítulo).

En el AFPP es muy precisa la alusión a la erradicación del TI, ya que es una problemática que se ha evidenciado en las actuaciones de las FARC, como una de las primeras entidades en la que los NNA no solo ejercieron el TI, sino que se llegó a los abusos de la EI (Rausky, 2009).

La EI ha dejado a cientos de niños absolutamente vulnerados en cuanto a sus derechos básicos (Pinzón et al., 2003), y el nivel de afectación es diferenciado. En algunos casos, la afectación ha sido grave y en otros casos es relativo, por lo que cada caso debe evaluarse en forma especial (Gómez, 2007).

Unas de las partes más importantes en el AFPP es el protocolo de monitoreo y modificación, mediante el cual se llevará a cabo un proceso de vigilancia y de decisión sobre las acciones a realizar o no respecto a los acuerdos ya impuestos (Jiménez-Peña, 2014). En este protocolo se menciona el cuidado de la apariencia personal y la buena presentación por parte de los exintegrantes de las FARC, la relación que tendrán con la población tanto afectada como no 
afectada (con el máximo respeto, cortesía y consideración), de forma que se denunciará cualquier acto o amenaza de explotación, violencia y abuso sexual, entre otras.

Este compromiso de denuncia está directamente conectado con el compromiso de no cometer ningún acto de violencia de género, incluida la explotación o abusos sexuales (Camacho-Ordóńez y Trujillo-González, 2009; Jiménez Villarreal y Jiménez Montalvo, 2019), tema absolutamente importante para asegurar el cumplimiento del acuerdo en materia de EI y de TI como principales problemáticas de vulneración de derechos de los NNA.

\section{1 ¿Que está pasando actualmente en materia de EI y TI?}

En la actualidad, particularmente en Colombia, el TI es notorio. Este no siempre se da en forma de vulneración de los derechos de los NNA, pero sí se evidencia que este grupo etario hace presencia en establecimientos de comercio para realizar labores básicas como limpieza, atención al público y otras tareas, incluso, en las calles de las ciudades (Martínez, 2001).

Pero últimamente reapareció otra forma de utilización de NNA la cual se centra en la EI. Esta consiste en el alquiler de niños que no están con sus padres, para generar un nivel más elevado de lástima a través de acciones de mendicidad. Para iniciar, se demuestra que muchos de los niños que están cargados suelen estar dormidos, pero la práctica verdadera es doparlos con drogas psiquiátricas que por lo general son la levomepromazina y la sinogán.

Dicho lo anterior, se patentiza la vulneración de un derecho fundamental, el derecho a la salud, ya que dichas drogas psiquiátricas pueden generar graves problemas en la salud de los infantes (Vasques et al., 2004) si son suministradas con mucha frecuencia. Esta práctica es llevada a cabo principalmente para que los NNA no interrumpan ni causen molestia a las personas a las que se les ofrece el producto a vender — que por lo general son dulces, esferos, bebidas, películas, música en discos compactos e implementos de aseo, entre otros-.

El negocio del alquiler vinculado a la mendicidad consta de varias características:

a. El "alquiler" de NNA presenta distintas tarifas que varían según la edad, la zona de acción, el rango de los tiempos en los cuales 
se desarrolla la actividad del menor o las características de este. El "negocio" resulta rentable para quien desarrolla la EI.

b. A las personas que los alquilan no les importa el derecho a la integridad personal de los menores, la cual puede verse socavada por otras personas con terceras intenciones o por la exposición a los fenómenos naturales como la lluvia y el sol, entre otras (Usera, 2006).

Detallamos este punto en particular, porque a raíz de la investigación hemos podido identificar una situación muy negativa en los NNA sometidos a estas prácticas de EI, principalmente a manos de sus propios progenitores, quienes exponen algunas condiciones que consideran válidas para su actuar, como estas:

a. La migración generada por el desplazamiento forzoso provocado por las acciones de los grupos insurgentes: cuando una familia se traslada a otro ámbito geográfico, ante la insuficiencia de medios económicos, los progenitores obligan a sus hijos a llevar a cabo actividades de mendicidad.

b. Las situaciones de división familiar, sea por abandono material de un progenitor o por la muerte de alguno de los padres.

c. La necesidad de tener un acceso a una rentabilidad económica sostenible. Variable que justifica el que las familias incorporen a sus hijas a actividades vinculadas a la prostitución o a la comercialización de estupefacientes. En el caso de los varones, la inserción en las actividades delincuenciales responde a la necesidad de los progenitores de contar con elementos que le faciliten el desarrollo de sus actividades ilícitas.

\subsection{Posibilidades de erradicación del TI}

Como principal estrategia para erradicar la EI y el TI se encuentran las campańas y fundaciones creadas con este objetivo, entre las cuales se destacan las siguientes:

a. Campaña "Tarjeta roja al trabajo infantil”: Como parte de la campaña del Día Mundial contra el Trabajo Infantil de la OIT, se lleva 
adelante la campaña de sensibilización “Tarjeta Roja”, para evidenciar que el TI es algo inaceptable y que atenta contra el bienestar de niños, niñas y adolescentes, perjudica su educación, su desarrollo y su sustento. La exposición temprana de niños y niñas al trabajo hace más difícil de alcanzar el objetivo del trabajo decente para todos y de la construcción de una sociedad de bienestar, productiva, digna e inclusiva.

b. Iniciativa "La música contra el trabajo infantil": Esta campaña fue lanzada en el 2018 por la OIT en compañía de algunos de los mejores músicos del mundo, a quienes se les pide que dediquen un concierto o una canción a esta causa. La educación musical empodera a los niños, desarrolla sus competencias y, esencialmente, los empuja a ir a la escuela y no abandonarla. Los socios de la iniciativa hacen un llamado a todos los actores del mundo de la música para que se unan al grupo en la sensibilización sobre el trabajo infantil y sobre la importancia de la educación musical y artística en la lucha contra este flagelo.

c. Scream: Defensa de los derechos de los niños a través de la educación, las artes y los medios de comunicación. Este programa tiene como objetivo hacer que estos jóvenes tomen conciencia de los derechos de los niños, con una atención particular sobre el trabajo infantil, para que ellos puedan a su vez difundir el mensaje y movilizar sus comunidades a que tomen acción. En definitiva, el programa Scream busca hacer cambiar las actitudes sociales con el fin de promover una cultura de respeto de los derechos de los niños y de fortalecer el movimiento mundial contra el trabajo infantil.

d. Jóvenes en acción contra el trabajo infantil: Mundialmente, los jóvenes denuncian el trabajo infantil y toman acciones con el fin de sensibilizar sobre este proceso, dándolo a conocer a los jóvenes de su edad, actuando como portavoces de aquellos niños cuyos derechos no se respetan y exigiendo a los responsables la toma de decisiones y la actuación urgente para proteger a los nińos que se encuentran en peligro (OIT, 2019). 
Finalmente, se evidencia que la problemática de TI y EI lleva largo tiempo atormentando a cientos de niños y vulnerando sus derechos fundamentales, a pesar de la existencia de medidas cautelares, leyes y artículos, entre otros, para proteger sus derechos, determinados como los más importantes frente a los demás.

\section{Conclusiones}

La problemática del conflicto armado en Colombia es un fuerte detonante para el no reconocimiento de los derechos de NNA, en especial, en los casos derivados de las acciones de los grupos armados que explotaban a esta población vulnerable para la satisfacción indiscriminada de sus intereses.

En el AFPP, los derechos de NNA son un tema primordial, no obstante, a pesar de que existen acuerdos en los que se pretende garantizar los derechos de estos, sumados a las campañas para la erradicación del TI, es evidente que no existe un castigo exacto para aquellas personas que explotan ni una reparación para las víctimas. Se concluye, por lo tanto, que siguen pasando los días y el tiempo y hay una gran cantidad de NNA cuyos derechos están siendo vulnerados, porque no se toman medidas penales ni acciones sociales contra la explotación laboral, sexual y mental de estos.

\section{Referencias}

Acevedo Suárez, A., y Rojas Castillo, Z. M. (2016). Generalidades del conflicto, los procesos de paz y el posconflicto. Revista de la Facultad de Derecho y Ciencias Políticas, 46 (124), 33-45. Bermúdez-Tapia, M. (2007). La Constitución a través de las sentencias del Tribunal Constitucional. Ediciones Legales.

Bermúdez-Tapia, M. (2012). Derecho procesal de familia. Editorial San Marcos.

Bermúdez-Tapia, M. (2018). Legitimidad de la legislación en el Estado de Derecho. En A. Martínez Lazcano, y A. Islas Colín (Eds.), Derechos humanos: la transformación de la cultura jurídica. Ediciones Nueva Jurídica

Bermúdez-Tapia, M. (2019). Análisis del perfil criminológico de adolescentes infractores para evaluar tasas de reincidencia delictiva que afecte la seguridad ciudadana. [Documento de trabajo]. UPSJB.

Bohórquez, L. M. (2009). El trabajo infantil en Colombia. Obtenido de Ministerio de Salud y Protección Social: https://www.minsalud.gov.co/Documents/NOTICIAS\%20 
OFICINA\%20COMUNICACIONES/Trabajo\%20y\%20Empleo/CIFRAS\%20\%20 ERRADICACI\%C3\%93N\%20DEL\%20TRABAJO\%20INFANTIL.pdf

Cabrera Cabrera, L. J., Corcione Nieto, M. A., Figueroa Pedreros, E. C. y Rodríguez Macea, C. D. (2018). Sobre relatos y memoria. Revista Cientifica General José María Córdova, 16(24), 177-201. https://doi.org/10.21830/19006586.363

Camacho Ordónez, L. B., y Trujillo González, M. A. (2009). La explotación sexual comercial infantil: una ganancia subjetiva. Revista Latinoamericana de Ciencias Sociales, Niñez y Juventud, 7(2), 1009-1025.

Cárdenas, J. D. (2013). Opinión pública y proceso de paz: actitudes e imaginarios de los bogotanos frente al proceso de paz de La Habana entre el gobierno colombiano y la guerrilla de las FARC. Ciudad Paz-Ando, 6(1), 41-58. https://doi.org/10.14483/udistrital.jour. cpaz.2013.1.a03

Castro Torres, I. E., Casallas Torres, D. F., y Salgado Torres, A. K. (2019). Representación social del Ejército Nacional en niños y niñas en condición vulnerable de Bogotá. Revista Científica General José María Córdova, 17(26), 327-355. https://doi.org/10.21830/19006586.405

Cillero, M. (1999). El interés superior del niño en el marco de la Convención Internacional sobre los Derechos del Niño. Justicia y Derechos del Niño número, 125, 1-16.

Contreras, M. H. J. (2003). El conflicto armado en Colombia. Revista de Derecho, (19) 119125. Recuperado de http://www.redalyc.org/articulo.oa?id=85101907

Corcione Nieto, M. A., Fernández-Osorio, A. E., Cabrera-Cabrera, L. J., y Rojas Yaima, B. C. (2019). Aportes de la Academia a la legitimidad de la justicia en Colombia. Revista Cientifica General José María Córdova, 17(28), 819-843. https://doi.org/10.21830/19006586.516

Cubides Cárdenas, J. A., Sierra Zamora, P. A., Calixto Ortiz, D. A., y Pabón Caballero, N. (2019). Terrorismo por parte de las FARC-EP y políticas públicas orientadas a la seguridad nacional en Colombia durante 1990-2000. Revista Cientifica General José María Córdova, 17(26), 309-325. https://doi.org/10.21830/19006586.404

CSI (2008). Trabajo Infantil. Confederación Sindical Internacional. Bélgica. https://www. ituc-csi.org/IMG/pdf/mini_guide_CL_final_ES.pdf

FARC-EP. (2019). Programa Agrario de los Guerrilleros. https://www.farc-ep.co/octava-conferencia/programa-agrario-de-los-guerrilleros-de-las-farc-ep.html

Fernández-Osorio, A. E. (2019). La implementación del Acuerdo de Paz con las FARC-EP: un estudio comparado desde la experiencia internacional. Análisis Político, 32(95), 104-124. https://doi.org/10.15446/anpol.v32n95.80977

Fernández-Osorio, A., y Pachon Pinzon, R. (2019). Reconciliation perspectives in Colombia: characterizing the 2016 peace agreement with the FARC. Revista Relaciones Internacionales, Estrategia y Seguridad, 14(1), 31-56. https://doi.org/10.18359/ries.3356

Gaitán, L. (2006). El bienestar social de la infancia y los derechos de los niños. Política y sociedad, 43(1), 63-80.

Gálvez, Í. Á. (2014). Universales, absolutos e inalienables: los derechos indestructibles. Revista de Humanidades de Valparaiso, (4), 63-80. 
Garay Acevedo, C. P., y Pérez Guecha, A. del P. (2018). Los derechos humanos en Colombia. Revista Cientifica General José María Córdova, 16(23), 83-105. https://doi. org/10.21830/19006586.307

Gómez, Z. P. (2007). El trabajo infantil en clave colonial: consideraciones histórico-antropológicas. Nómadas, 26, 80-90.

Jiménez-Peña, G. (2014). Multinacionales y responsabilidad social empresarial en la construcción de paz en Colombia. Cuadernos de Administración, 27(48), 67-96. https://doi. org/10.11144/Javeriana.cao48-27.mrse

Jiménez Villarreal, J. E., y Jiménez Montalvo, D. A. (2019). Violencia sexual en zonas de posconflicto. Revista Cientifica General José María Córdova, 17(27), 505-523. https://doi. org/10.21830/19006586.436

Martínez, A. M. (2001). Trabajo infantil y subjetividad: una perspectiva necesaria. Estudios de Psicología, 6(2), 235-244. http://dx.doi.org/10.1590/S1413-294X2001000200011

Nasi, C. y Rettberg, A. (2005). Los estudios sobre conflicto armado y paz: un campo en evolución permanente. Colombia Internacional, 62, 64-85. https://doi.org/10.7440/colombiaint62.2005.04

Niño González, C. A. (2016). El narcotráfico mutante: nueva perspectiva de análisis del fenómeno en Colombia. Revista Cientifica General José María Córdova, 14(18), 113-124. https://doi.org/10.21830/19006586.45

Novoa, D. P. S. (2017). El sistema pensional colombiano: desafíos después de la reforma y re-reformas. El Cotidiano, 204, 128-135.

Oficina del Alto Comisionado para la Paz. (2016). Acuerdo final para la terminación del conflicto y la construcción de una paz estable y duradera. http://www.altocomisionadoparalapaz.gov. co/procesos-y-conversaciones/acuerdo-general/Paginas/inicio.aspx

OIT y IPEC (2003). Tercer Plan Nacional para la Erradicación del Trabajo Infantil y la Protección del Trabajo Juvenil en Colombia, 2003-2006. Colombia: Organización Internacional del Trabajo. http://www.ilo.org/ipec/Informationresources/WCMS_IPEC_PUB_7493/ lang--es/index.htm

Organización Internacional del Trabajo. (2001). Convenio sobre las Peores Formas de Trabajo Infantil. https://www.ilo.org/dyn/normlex/es/f?p=1000:13100:0::NO:13100:P13100_ COMMENT_ID:2243535

Pautassi, L. C., y Zibecchi, C. (2010). La provisión de cuidado y la superación de la pobreza infantil. Programas de transferencias condicionadas en Argentina y el papel de las organizaciones sociales y comunitarias. Cepal.

Peña Chivatá, C., Sierra Zamora, P. A., y Hoyos Rojas, J. C. (2019). La política de fronteras de Colombia ante las nuevas amenazas de seguridad y defensa. Revista Cientifica General José María Córdova, 17(28), 773-795. https://doi.org/10.21830/19006586.473

Pinzón, Á. M., Briceño, L., Gómez, A. I., y Latorre, C. (2003). Trabajo infantil en las calles de Bogotá. Revista Ciencias de la Salud, 1(2), 151-163.

Rausky, M. E. (2009). ¿ ¿nfancia sin trabajo o infancia trabajadora? Perspectivas sobre el trabajo infantil. Revista Latinoamericana de Ciencias Sociales, Niñez y Juventud, 7 (2), 681-706. 
Roldán Ruiz, J. (2002). Conversaciones cruzadas: otras voces hablan del arte como una posible herramienta para la construcción de paz. https://repository.javeriana.edu.co/handle/10554/20178

Ruiz, J. I. (2012). Metodología de la investigación cualitativa (Vol. 15). Universidad de Deusto.

Sánchez, S. (2019). Asi ha sido la Colombia de las FARC: más de 7 millones de víctimas en 52 años. Magnet.xataka.com. https://magnet.xataka.com/preguntas-no-tan-frecuentes/asi-ha-sido-la-colombia-de-las-farc-mas-de-7-millones-de-victimas-en-52-anos

Soto, M. (2012). Trabajo infantil. F. Romani Allpacca, Entrevistador.

Springer, N. (2012). Como corderos entre lobos. Del uso y reclutamiento de niñas, niños y adolescentes en el marco del conflicto armado y la criminalidad en Colombia. Centro de Memoria Histórica.

Usera, R. C. (2006). El derecho a la integridad personal. Lex Nova.

Valencia, G. D., Gutiérrez Loaiza, A., y Johansson, S. (2012). Negociar la paz: una síntesis de los estudios sobre la resolución negociada de conflictos armados internos. Estudios Políticos, 40, 149-174.

Vasques, F., Martins, F. C., y Pinto de Azevedo, A. (2004). Aspectos psiquiátricos do tratamento da obesidade. Archives of Clinical Psychiatry, 31(4), 195-198. http://dx.doi.org/10.1590/ S0101-60832004000400013 